\title{
Approximation of Common Fixed Points for a Family of Non- Lipschitzian Mappings
}

\author{
TAE HWA KIM* \\ Division of Mathematical Sciences, Pukyong National University, Busan 608-737, \\ Korea \\ e-mail : taehwa@pknu.ac.kr \\ YONG KIL PARK \\ Department of Liberal Arts, Hanzhong University, Donghae 240-713, Korea \\ e-mail : ykpark@hanzhong.ac.kr

\begin{abstract}
In this paper, we first introduce a family $\mathcal{S}=\left\{S_{n}: C \rightarrow C\right\}$ of non-Lipschitzian mappings, called total asymptotically nonexpansive (briefly, TAN) on a nonempty closed convex subset $C$ of a real Banach space $X$, and next give necessary and sufficient conditions for strong convergence of the sequence $\left\{x_{n}\right\}$ defined recursively by the algorithm $x_{n+1}=S_{n} x_{n}, n \geq 1$, starting from an initial guess $x_{1} \in C$, to a common fixed point for such a continuous TAN family $\mathcal{S}$ in Banach spaces. Finally, some applications to a finite family of TAN self mappings are also added.
\end{abstract}

\section{Introduction}

Let $C$ be a nonempty closed convex subset of a real Banach space $X$ and let $T: C \rightarrow C$ be a mapping. Then $T$ is said to be a Lipschitzian mapping if, for each $n \geq 1$, there exists a constant $k_{n}>0$ such that

$$
\left\|T^{n} x-T^{n} y\right\| \leq k_{n}\|x-y\|
$$

for all $x, y \in C$ (we may assume that all $k_{n} \geq 1$ ). A Lipschitzian mapping $T$ is called uniformly $k$-Lipschitzian if $k_{n}=k$ for all $n \geq 1$, nonexpansive if $k_{n}=1$ for all $n \geq 1$, and asymptotically nonexpansive [4] if $\lim _{n \rightarrow \infty} k_{n}=1$, respectively. The class of asymptotically nonexpansive mappings was introduced by Goebel and Kirk [4] as a generalization of the class of nonexpansive mappings. They proved that if $C$ is a nonempty bounded closed convex subset of a uniformly convex Banach space $X$, then every asymptotically nonexpanisve mapping $T: C \rightarrow C$ has a fixed point.

On the other hand, as the classes of non-Lipschitzian mappings, there appear in the literature two definitions, one is due to Kirk who says that $T$ is a mapping

* Corresponding Author.

Received September 25, 2009; accepted November 16, 2009.

2000 Mathematics Subject Classification: Primary 47H09, Secondary 65J15.

Key words and phrases: Common fixed points, non-Lipschitzian mappings, total asymptotically nonexpansive mappings, strong convergence. 
of asymptotically nonexpansive type [7] if for each $x \in C$,

$$
\limsup _{n \rightarrow \infty} \sup _{y \in C}\left(\left\|T^{n} x-T^{n} y\right\|-\|x-y\|\right) \leq 0
$$

and $T^{N}$ is continuous for some $N \geq 1$. The other is the stronger concept due to Bruck, Kuczumov and Reich [2]. They say that $T$ is asymptotically nonexpansive in the intermediate sense if $T$ is uniformly continuous and

$$
\limsup _{n \rightarrow \infty} \sup _{x, y \in C}\left(\left\|T^{n} x-T^{n} y\right\|-\|x-y\|\right) \leq 0
$$

In this case, observe that if we define

$$
\delta_{n}:=\sup _{x, y \in C}\left(\left\|T^{n} x-T^{n} y\right\|-\|x-y\|\right) \vee 0,
$$

where $a \vee b:=\max \{a, b\}$, then $\delta_{n} \rightarrow 0$ and (1.3) immediately reduces to

$$
\left\|T^{n} x-T^{n} y\right\|-\|x-y\| \leq\|x-y\|+\delta_{n}
$$

for all $x, y \in C$ and $n \geq 1$.

Recently, Alber et al. [1] introduced the wider class of total asymptotically nonexpansive mappings to unify various definitions of classes of nonlinear mappings associated with the class of asymptotically nonexpansive mappings; see also Definition 1 of [3]. They say that a mapping $T: C \rightarrow C$ is said to be total asymptotically nonexpansive (TAN, in brief) [1] if there exist sequences $\left\{c_{n}\right\}$ and $\left\{d_{n}\right\}$ of nonnegative real numbers with $c_{n}, d_{n} \rightarrow 0$ as $n \rightarrow \infty$ and $\phi \in \Gamma\left(\mathbb{R}^{+}\right)$such that

$$
\left\|T^{n} x-T^{n} y\right\| \leq\|x-y\|+c_{n} \phi(\|x-y\|)+d_{n},
$$

for all $x, y \in C$ and $n \geq 1$, where $\mathbb{R}^{+}:=[0, \infty)$ and

$\phi \in \Gamma\left(\mathbb{R}^{+}\right) \Leftrightarrow \phi$ is strictly increasing, continuous on $\mathbb{R}^{+}$and $\phi(0)=0$.

Remark 1.1. (i) Note that if $T$ is continuous, the property (1.5) with $c_{n}=0$ for all $n \geq 1$ is equivalent to (1.4) with $d_{n}=\delta_{n}$, and also that a mapping satisfying the property (1.3) is non-Lipschitzian; see [6].

(ii) Also, if we take $\phi(t)=t$ for all $t \geq 0$ and $d_{n}=0$ for all $n \geq 1$ in (1.5), it can be reduced to the concept of asymptotically nonexpansive mapping. Furthermore, in addition, taking $c_{n}=0$ for all $n \geq 1$, it is nonexpansive, that is,

$$
\|T x-T y\| \leq\|x-y\|
$$

for all $x, y \in C$.

A point $x \in C$ is a fixed point of $T$ provided $T x=x$. Denote by $F(T):=\{x \in$ $C: T x=x\}$ the set of all fixed points of $T$. 
Let $\left\{T_{i}\right\}_{i=1}^{N}$ be a finite family of mappings from $C$ into itself. Then we denote $T_{n \bmod N}$ by $T_{[n]}$, namely, the $\bmod$ function takes values in the set $\{1,2, \cdots, N\}$ as

$$
T_{[n]}:= \begin{cases}T_{N}, & \text { if } r=0 \\ T_{r}, & \text { if } 0<r<N\end{cases}
$$

for $n=k N+r$ for some integers $j \geq 0$ and $0 \leq r<N$. In this case, setting

$$
k(n):= \begin{cases}k, & \text { if } r=0 \\ k+1, & \text { if } 0<r<N\end{cases}
$$

for each $n \geq 1$, it is not hard to see that $k(n) \rightarrow \infty$ as $n \rightarrow \infty$,

$$
k(n-N)=k(n)-1, \text { and } T_{[n-N]}=T_{[n]}, \quad n \geq N .
$$

We begin with the following simple observation.

Proposition 1.2. Let $C$ be a nonempty closed convex subset of a real Banach space $X, N \geq 1$ a positive integer, and let $\left\{T_{i}\right\}_{i=1}^{N}$ be a finite family of TAN mappings from $C$ into itself. Then there exist sequences $\left\{c_{n}\right\}$ and $\left\{d_{n}\right\}$ of nonnegative real numbers with $c_{n}, d_{n} \rightarrow 0$ and $\phi \in \Gamma\left(\mathbb{R}^{+}\right)$such that

$$
\left\|A_{n} x-A_{n} y\right\| \leq\|x-y\|+c_{n} \phi(\|x-y\|)+d_{n},
$$

for all $x, y \in C$ and $n \geq 1$, where

$$
A_{n} \text { is either } T_{[n]}^{n} \text { or } \sum_{i=1}^{N} \lambda_{i}^{(n)} T_{i}^{(n)} \text {, }
$$

for all the $\lambda_{i}^{(n)} \in[0,1]$ with $\sum_{i=1}^{N} \lambda_{i}^{(n)}=1$. In particular, if $k(n)$ is given as in (1.6), then

$$
\left\|T_{[n]}^{k(n)} x-T_{[n]}^{k(n)} y\right\| \leq\|x-y\|+c_{n} \phi(\|x-y\|)+d_{n} .
$$

Definition 1.3. Let $C$ be a nonempty closed convex subset of a real Banach space $X$. A discrete family $\mathcal{S}=\left\{S_{n}: C \rightarrow C\right\}$ is said to be TAN on $C$ if there exist sequences $\left\{c_{n}\right\}$ and $\left\{d_{n}\right\}$ of nonnegative real numbers converging to zero and $\phi \in \Gamma\left(\mathbb{R}^{+}\right)$such that

$$
\left\|S_{n} x-S_{n} y\right\| \leq\|x-y\|+c_{n} \phi(\|x-y\|)+d_{n}
$$

for all $x, y \in C$ and $n \geq 1$. Furthermore, we say that $\mathcal{S}$ is continuous on $C$ provided each $S_{n} \in \mathcal{S}$ is continuous on $C$.

Example 1.4. The discrete families of $\left\{A_{n}\right\}_{n=1}^{\infty}$ and $\left\{T_{[n]}^{k(n)}\right\}_{n=1}^{\infty}$ in Proposition 1.2 are obviously TAN on $C$. 
Example 1.5. Let $X=\mathbb{R}, C=[0, \infty)$ and, for each $n \geq 1$, define

$$
S_{n} x=\left(1+\frac{1}{n}\right) x+\frac{1}{n} \tan ^{-1} x, \quad x \in C .
$$

Then the family $\mathcal{S}=\left\{S_{n}: C \rightarrow C\right\}$ is continuous TAN on $C$. In fact, use $\left|\tan ^{-1} x\right|<$ $\frac{\pi}{2}$ to get

$$
\left|S_{n} x-S_{n} y\right| \leq\left(1+\frac{1}{n}\right)|x-y|+\frac{\pi}{n}
$$

for all $x, y \in C$ and $n \geq 1$, where $\phi(t)=t, c_{n}=\frac{1}{n}$ and $d_{n}=\frac{\pi}{n}$.

Moreover, we have the following

Example 1.6. Let $C$ be a nonempty closed convex subset of a real Banach space $X$. Let two families $\Im_{i}=\left\{T_{n}^{(i)}: C \rightarrow C\right\}$ be continuous TAN on $C$ satisfying the property (1.8) with $c_{n}^{(i)} \in[0,1], d_{n}^{(i)} \equiv 0$ and $\phi_{i} \in \Gamma\left(\mathbb{R}^{+}\right)$for $i=1,2$, respectively. Let $\left\{\alpha_{n}\right\}$ and $\left\{\beta_{n}\right\}$ be sequences in $[0,1]$. Then the family $\mathcal{S}=\left\{S_{n}: C \rightarrow C\right\}$ defined by

$$
S_{n}=\left(1-\alpha_{n}\right) I+\alpha_{n} T_{n}^{(1)}\left[\left(1-\beta_{n}\right) I+\beta_{n} T_{n}^{(2)}\right]
$$

for each $n \geq 1$ is also continuous TAN on $C$.

Proof. Putting $U_{n}:=\left(1-\beta_{n}\right) I+\beta_{n} T_{n}^{(2)}$ and using (1.8) yield

$$
\begin{aligned}
\left\|U_{n} x-U_{n} y\right\| & \leq\left(1-\beta_{n}\right)\|x-y\|+\beta_{n}\left\|T_{n}^{(2)} x-T_{n}^{(2)} y\right\| \\
& \leq\left(1-\beta_{n}\right)\|x-y\|+\beta_{n}\left[\|x-y\|+c_{n}^{(2)} \phi_{2}(\|x-y\|)\right] \\
& \leq\|x-y\|+c_{n}^{(2)} \phi_{2}(\|x-y\|)
\end{aligned}
$$

for all $x, y \in C$. Then, we can also compute

$$
\begin{aligned}
\left\|S_{n} x-S_{n} y\right\| & \leq\left(1-\alpha_{n}\right)\|x-y\|+\alpha_{n}\left\|T_{n}^{(1)}\left(U_{n} x\right)-T_{n}^{(1)}\left(U_{n} y\right)\right\| \\
& \leq\left(1-\alpha_{n}\right)\|x-y\|+\alpha_{n}\left[\left\|U_{n} x-U_{n} y\right\|+c_{n}^{(1)} \phi_{1}\left(\left\|U_{n} x-U_{n} y\right\|\right)\right] \\
& \leq\|x-y\|+c_{n}^{(2)} \phi_{2}(\|x-y\|)+c_{n}^{(1)} \phi_{1}\left(\|x-y\|+M \phi_{2}(\|x-y\|)\right) \\
& =\|x-y\|+c_{n} \psi(\|x-y\|),
\end{aligned}
$$

where $\psi(t):=\phi_{2}(t)+\phi_{1}\left(t+M \phi_{2}(t)\right)$ for all $t \geq 0, M:=\sup _{n \geq 1} c_{n}^{(2)}$, and $c_{n}:=$ $\max \left\{c_{n}^{(1)}, c_{n}^{(2)}\right\}$. Therefore, the family $\mathcal{S}=\left\{S_{n}: C \rightarrow C\right\}$ is continuous TAN on $C$ with $c_{n}$ and $\psi \in \Gamma\left(\mathbb{R}^{+}\right)$.

Example 1.7. Let $C$ be a nonempty closed convex subset of a real Banach space $X$. Let families $\Im_{i}=\left\{T_{n}^{(i)}: C \rightarrow C\right\}$ be continuous TAN on $C$, equipped with $c_{n}^{(i)} \in[0,1], d_{n}^{(i)}$ and $\phi_{i} \in \Gamma\left(\mathbb{R}^{+}\right)$as in (1.8) for $i=1,2, \cdots, N$, respectively, such that the following two properties hold:

$(\widetilde{C} 1) \exists \alpha_{0}, \beta>0$ such that $\phi_{i}(t) \leq \alpha_{0} t$ for all $t \geq \beta, 1 \leq i \leq N$; 
$(\widetilde{C} 2) \sum_{n=1}^{\infty} c_{n}^{(i)}<\infty$ and $\sum_{n=1}^{\infty} d_{n}^{(i)}<\infty, 1 \leq i \leq N$.

Let $\left\{\alpha_{n}^{(i)}\right\}$ be sequences in $[0,1]$ for $1 \leq i \leq N$. Then the family $\mathcal{S}=\left\{S_{n}: C \rightarrow C\right\}$ defined by

$$
\begin{aligned}
S_{n}= & \left(1-\alpha_{n}^{(1)}\right) I+\alpha_{n}^{(1)} T_{n}^{(1)}\left[\left(1-\alpha_{n}^{(2)}\right) I+\alpha_{n}^{(2)} T_{n}^{(2)}\left[\left(1-\alpha_{n}^{(3)}\right) I+\right.\right. \\
& \left.\alpha_{n}^{(3)} T_{n}^{(3)}\left(\cdots+\alpha_{n}^{(N-1)} T_{n}^{(N-1)}\left(\left(1-\alpha_{n}^{(N)}\right) I+\alpha_{n}^{(N)} T_{n}^{(N)}\right) \cdots\right)\right]
\end{aligned}
$$

is also continuous TAN on $C$, namely, there exist $\left\{c_{n}\right\},\left\{d_{n}\right\}$ and $\phi \in \Gamma\left(\mathbb{R}^{+}\right)$such that

$$
\left\|S_{n} x-S_{n} y\right\| \leq\|x-y\|+c_{n} \phi(\|x-y\|)+d_{n}, \quad x, y \in C .
$$

Furthermore, the following properties are also satisfied:

$(\widetilde{C} 1)^{\prime} \exists \alpha\left(\geq \alpha_{0}\right), \beta>0$ such that $\phi(t) \leq \alpha t$ for all $t \geq \beta$.

$(\widetilde{C} 2)^{\prime} \quad \sum_{n=1}^{\infty} c_{n}<\infty$ and $\sum_{n=1}^{\infty} d_{n}<\infty$.

Proof. First, we claim that the family $\mathcal{S}=\left\{S_{n}: C \rightarrow C\right\}$ is continuous TAN on $C$ for $N=2$, that is, where

$$
S_{n}=\left(1-\alpha_{n}^{(1)}\right) I+\alpha_{n}^{(1)} T_{n}^{(1)}\left[\left(1-\alpha_{n}^{(2)}\right) I+\alpha_{n}^{(2)} T_{n}^{(2)}\right] .
$$

Indeed, putting $U_{n}:=\left(1-\alpha_{n}^{(2)}\right) I+\alpha_{n}^{(2)} T_{n}^{(2)}$ simply and using (1.8) yield

$$
\begin{aligned}
\left\|U_{n} x-U_{n} y\right\| & \leq\left(1-\alpha_{n}^{(2)}\right)\|x-y\|+\alpha_{n}^{(2)}\left\|T_{n}^{(2)} x-T_{n}^{(2)} y\right\| \\
& \left.\leq\left(1-\alpha_{n}^{(2)}\right)\|x-y\|+\alpha_{n}^{(2)}\left[\|x-y\|+c_{n}^{(2)} \phi_{2}(\|x-y\|)\right]+d_{n}^{(2)}\right] \\
& \leq\|x-y\|+c_{n}^{(2)} \phi_{2}(\|x-y\|)+d_{n}^{(2)}
\end{aligned}
$$

for all $x, y \in C$. Then, we can also have

$$
\begin{aligned}
(1.10)\left\|S_{n} x-S_{n} y\right\| \leq & \left(1-\alpha_{n}^{(1)}\right)\|x-y\|+\alpha_{n}^{(1)}\left\|T_{n}^{(1)}\left(U_{n} x\right)-T_{n}^{(1)}\left(U_{n} y\right)\right\| \\
\leq & \left(1-\alpha_{n}^{(1)}\right)\|x-y\|+\alpha_{n}^{(1)}\left[\left\|U_{n} x-U_{n} y\right\|\right. \\
& \left.+c_{n}^{(1)} \phi_{1}\left(\left\|U_{n} x-U_{n} y\right\|\right)+d_{n}^{(1)}\right] .
\end{aligned}
$$

Using $(\widetilde{C} 1)$ and the strictly increasing property of $\phi_{i}$, we easily see

$$
\phi_{i}(t) \leq \phi_{i}(\beta)+\alpha t
$$

for all $t \geq 0$ and $1 \leq i \leq N$. In particular,

$$
\phi_{1}\left(\left\|U_{n} x-U_{n} y\right\|\right) \leq \phi_{1}(\beta)+\alpha\left\|U_{n} x-U_{n} y\right\| .
$$

Now substituting (1.11) combined with (1.9) into (1.10) and simplifying, we get

$$
\begin{aligned}
\left\|S_{n} x-S_{n} y\right\| \leq & \|x-y\|+c_{n}^{(2)} \phi_{2}(\|x-y\|)+c_{n}^{(1)}\left[\alpha\left(\|x-y\|+c_{n}^{(2)} \phi_{2}(\|x-y\|)\right]\right. \\
& +d_{n}^{(1)}+\left(1+\alpha c_{n}^{(1)}\right) d_{n}^{(2)}+\phi_{1}(\beta) c_{n}^{(1)} \\
\leq & \|x-y\|+c_{n} \psi(\|x-y\|)+d_{n},
\end{aligned}
$$


where $\phi(t):=\phi_{2}(t)+\phi_{1}\left(\alpha\left(t+M \phi_{2}(t)\right)\right)$ for all $t \geq 0, M:=\sup _{n>1} c_{n}^{(2)}, c_{n}:=$ $\max \left\{c_{n}^{(1)}, c_{n}^{(2)}\right\}$ and $d_{n}:=d_{n}^{(1)}+\left(1+\alpha c_{n}^{(1)}\right) d_{n}^{(2)}+\phi_{1}(\beta) c_{n}^{(1)}$. Therefore, the family $\mathcal{S}=\left\{S_{n}: C \rightarrow C\right\}$ is continuous TAN on $C$ with $c_{n}, d_{n}$ and $\phi \in \Gamma\left(\mathbb{R}^{+}\right)$for $N=2$. Obviously,

$$
\begin{aligned}
\phi(t) & =\phi_{2}(t)+\phi_{1}\left(\alpha\left(t+M \phi_{2}(t)\right)\right) \\
& \leq \alpha\left[t+\alpha\left(t+M \phi_{2}(t)\right)\right] \\
& \leq \alpha(1+\alpha+M) t:=\tilde{\alpha} t, \quad t \geq \beta
\end{aligned}
$$

and also $\sum_{n=1}^{\infty} c_{n}<\infty, \sum_{n=1}^{\infty} d_{n}<\infty$.

Now use the mathematical induction to complete the proof.

Remark 1.8. Note that $S_{n}$ in Example 1.7 is rewritten as the following recursive form:

$$
\left\{\begin{array}{l}
S_{n}=\left(1-\alpha_{n}^{(1)}\right) I+\alpha_{n}^{(1)} T_{n}^{(1)} U_{n}^{(1)}, \\
U_{n}^{(1)}=\left(1-\alpha_{n}^{(2)}\right) I+\alpha_{n}^{(2)} T_{n}^{(2)} U_{n}^{(2)}, \\
\quad \vdots \\
U_{n}^{(N-2)}=\left(1-\alpha_{n}^{(N-1)}\right) I+\alpha_{n}^{(N-1)} T_{n}^{(N-1)} U_{n}^{(N-1)}, \\
U_{n}^{(N-1)}=\left(1-\alpha_{n}^{(N)}\right) I+\alpha_{n}^{(N)} T_{n}^{(N)}, \quad n \geq 1,
\end{array}\right.
$$

First let us consider a brief history of strong convergence problems for a single non-Lipschitzian mapping $T: C \rightarrow C$ which is both completely continuous and asymptotically nonexpansive in the intermediate sense as in (1.3) with $F(T) \neq \emptyset$.

Theorem 1.9([6]). Suppose that a mapping $T: C \rightarrow C$ is both completely continuous and $A N$ in the intermediate sense with $F(T) \neq \emptyset$. Let $\left\{x_{n}\right\}$ be a sequence defined by

$$
\left\{\begin{array}{l}
x_{1} \in C \text { chosen arbitrarily, } \\
x_{n+1}=\alpha_{n} x_{n}+\beta_{n} T^{n} y_{n}+\gamma_{n} u_{n}, \\
y_{n}=\alpha_{n}^{\prime} x_{n}+\beta_{n}^{\prime} T^{n} x_{n}+\gamma_{n}^{\prime} v_{n}, \quad n \geq 1,
\end{array}\right.
$$

where $\left\{\alpha_{n}\right\},\left\{\beta_{n}\right\},\left\{\gamma_{n}\right\},\left\{\alpha_{n}^{\prime}\right\},\left\{\beta_{n}^{\prime}\right\},\left\{\gamma_{n}^{\prime}\right\}$ are real sequences in [0,1] and $\left\{u_{n}\right\}_{n=1}^{\infty}$, $\left\{v_{n}\right\}_{n=1}^{\infty}$ are two bounded sequences in $C$ such that

(i) $\left\{\alpha_{n}\right\}$ is bounded away from $0,\left\{\beta_{n}^{\prime}\right\}$ is bounded away from 1 , and $\left\{\beta_{n}\right\}$ is bounded away from both 0 and 1 .

(ii) $\alpha_{n}+\beta_{n}+\gamma_{n}=\alpha_{n}^{\prime}+\beta_{n}^{\prime}+\gamma_{n}^{\prime}=1$ for all $n \geq 1$,

(iii) $\sum_{n=1}^{\infty} \gamma_{n}<\infty, \sum_{n=1}^{\infty} \gamma_{n}^{\prime}<\infty$ and $\sum_{n=1}^{\infty} \delta_{n}<\infty$, where $\delta_{n}$ is given as in (1.4). Then, $\left\{x_{n}\right\}$ converges strongly to a fixed point of $T$.

Recently, Chidume and Ofoedu [3] established the following necessary and sufficient condition for strong convergence for a finite family of TAN self mappings defined on a nonempty closed convex subset in real Banach spaces. 
Theorem 1.10([3]). Let $C$ be a nonempty closed convex subset of a real Banach space $X$ and let $\left\{T_{i}\right\}_{i=1}^{N}$ be a finite family of TAN mappings from $C$ into itself with $F_{N}:=\cap_{i=1}^{N} F\left(T_{i}\right) \neq \emptyset$. Let $\left\{x_{n}\right\}$ be the sequence defined explicitly by either

$$
\left\{\begin{array}{l}
x_{1} \in C \text { chosen arbitrarily, } \\
x_{n+1}=\left(1-\alpha_{n}\right) x_{n}+\alpha_{n} T_{1}^{n} x_{n}, \quad n \geq 1
\end{array}\right.
$$

for $N=1$ or

$$
\left\{\begin{array}{l}
x_{1} \in C \text { chosen arbitrarily, } \\
x_{n+1}=\left(1-\alpha_{n}\right) x_{n}+\alpha_{n} T_{1}^{n} y_{1 n}, \\
y_{1 n}=\left(1-\alpha_{n}\right) x_{n}+\alpha_{n} T_{2}^{n} y_{2 n}, \\
\quad \vdots \\
y_{(N-2) n}=\left(1-\alpha_{n}\right) x_{n}+\alpha_{n} T_{N-1}^{n} y_{(N-1) n}, \\
y_{(N-1) n}=\left(1-\alpha_{n}\right) x_{n}+\alpha_{n} T_{N}^{n} x_{n}, \quad n \geq 1
\end{array}\right.
$$

for $N \geq 2$, where $\left\{\alpha_{n}\right\}$ is a sequence in $[0,1]$. Assume that $\left\{c_{n}^{(i)}\right\},\left\{d_{n}^{(i)}\right\}$ and $\phi_{i}$, $1 \leq i \leq N$, satisfy the following properties:

(C1) $\exists \alpha_{i}, \beta_{i}>0$ such that $\phi_{i}(t) \leq \alpha_{i}$ t for all $t \geq \beta_{i}, 1 \leq i \leq N$.

(C2) $\sum_{n=1}^{\infty} c_{n}^{(i)}<\infty$ and $\sum_{n=1}^{\infty} d_{n}^{(i)}<\infty, 1 \leq i \leq N$;

Then $\left\{x_{n}\right\}$ converges strongly to a common fixed point of $\left\{T_{i}\right\}_{i=1}^{N}$ if and only if $\liminf _{n \rightarrow \infty} d\left(x_{n}, F_{N}\right)=0$, where $d(z, A):=\inf _{a \in A}\|x-a\|$ for all $z \in C$ and $A \subset C$.

Remark 1.11. (i) $\phi_{i}(t)=t^{s}, 0<s \leq 1,1 \leq i \leq N$ enjoys the condition (C1); see Remark 15 of [3].

(ii) For any fixed $\beta_{i}>0$ and $f \in \Gamma\left(\left[0, \beta_{i}\right)\right), 1 \leq i \leq N$, define a function $\phi_{i}$ by

$$
\phi_{i}(t)= \begin{cases}\int_{0}^{t} f(s) d s & \text { if } 0 \leq t<\beta_{i} \\ \frac{1}{\beta_{i}} \phi\left(\beta_{i}-\right) t & \text { if } t \geq \beta_{i}\end{cases}
$$

provided $\phi\left(\beta_{i}-\right):=\lim _{t \rightarrow \beta_{i}-} \phi(t)$ exists. Then, $\phi_{i} \in \Gamma\left(\mathbb{R}^{+}\right), 1 \leq i \leq N$ and it obviously satisfies the condition (C1) with $\alpha_{i}:=\frac{1}{\beta_{i}} \phi\left(\beta_{i}-\right)$. Especially, note that if $X$ is uniformly convex, taking $f:=\delta_{X}$, modulus of convexity of $X$, we see that (1.14) holds with $\beta_{i}:=2,1 \leq i \leq N$, because $\delta_{X}:[0,2] \rightarrow[0,1]$ is continuous on $[0,2), \delta_{X}(2)=1$, and strictly increasing on $[0,2]$; see [5] or [9].

(iii) Note that $(\mathrm{C} 1)$ is equivalent to $(\widetilde{C} 1)$. In fact, for $(\mathrm{C} 1) \Rightarrow(\widetilde{C} 1)$, take $\alpha:=$ $\max \left\{\alpha_{i}: 1 \leq i \leq N\right\}$ and $\beta:=\max \left\{\beta_{i}: 1 \leq i \leq N\right\}$. For the converse, take $\alpha_{i}=\alpha$ and $\beta_{i}=\beta$ for all $i=1,2, \cdots, N$.

In particular, taking $T_{n}^{(i)}=T_{i}^{n}, \alpha_{n}^{(i)}=\alpha_{n}$ in (1.13), by Example 1.7, the family $\mathcal{S}=\left\{S_{n}: C \rightarrow C\right\}$ defined by (1.12) is also continuous TAN on $C$. Therefore, the explicit algorithm (1.13) can be shortly rewritten as

$$
x_{n+1}=S_{n} x_{n}, \quad n \geq 1 .
$$


In this paper, motivated and stimulated by the result(see Theorem 1.10) by Chidume and Ofoedu [3], we shall give necessary and sufficient conditions for strong convergence of the algorithm (1.15) to a common fixed point for a continuous TAN family $\mathcal{S}=\left\{S_{n}: C \rightarrow C\right\}$ of non-Lipschitzian mappings, defined as in Definition 1.3. Also, some applications to a finite family of TAN self mappings are added.

\section{Necessary and sufficient conditions for convergence}

Theorem 2.1. Let $X$ be a real Banach space, $C$ be a nonempty closed convex subset of $X$. Let a discrete family $\mathcal{S}=\left\{S_{n}: C \rightarrow C\right\}$ be continuous TAN on $C$ with $F:=\cap_{n=1}^{\infty} F\left(S_{n}\right) \neq \emptyset$. Assume that $\left\{c_{n}\right\},\left\{d_{n}\right\}$ and $\phi$ satisfy the following two conditions:

(A1) $\exists \alpha, \beta>0$ such that $\phi(t) \leq \alpha$ for all $t \geq \beta$.

(A2) $\sum_{n=1}^{\infty} c_{n}<\infty, \sum_{n=1}^{\infty} d_{n}<\infty$.

Then the sequence $\left\{x_{n}\right\}$ defined by the explicit iteration method (1.15) converges strongly to a common fixed point of $\mathcal{S}$ if and only if $\liminf _{n \rightarrow \infty} d\left(x_{n}, F\right)=0$.

For the proof of Theorem 2.1, we shall need the following subsequent lemmas.

Lemma 2.2([8], [10]). Let $\left\{a_{n}\right\},\left\{\tilde{c}_{n}\right\}$ and $\left\{\tilde{d}_{n}\right\}$ be sequences of nonnegative real numbers such that

$$
a_{n+1} \leq\left(1+\tilde{c}_{n}\right) a_{n}+\tilde{d}_{n}
$$

for all $n \geq 1$. Suppose that $\sum_{n=1}^{\infty} \tilde{c}_{n}<\infty$ and $\sum_{n=1}^{\infty} \tilde{d}_{n}<\infty$. Then $\lim _{n \rightarrow \infty} a_{n}$ exists. Moreover, if in addition, $\liminf _{n \rightarrow \infty} a_{n}=0$, then $\lim _{n \rightarrow \infty} a_{n}=0$.

Lemma 2.3. Under the same hypotheses as Theorem 2.1, there hold the following properties:

(i) $\lim _{n \rightarrow \infty}\left\|x_{n}-p\right\|$ exists for all $p \in F$, and hence $\left\{x_{n}\right\}$ is bounded.

(ii) $\lim _{n \rightarrow \infty} d\left(x_{n}, F\right)$ exists.

Proof. First, to prove (i), let $p \in F$ and let $n \geq 1$ be arbitrarily given. Using (A1) and strict increasing of $\phi$, we easily get

$$
\phi(t) \leq \phi(\beta)+\alpha t, \quad t \geq 0 .
$$

Use (1.8) and (2.1) in turn to derive

$$
\begin{aligned}
\left\|x_{n+1}-p\right\| & =\left\|S_{n} x_{n}-S_{n} p\right\| \\
& \leq\left\|x_{n}-p\right\|+c_{n} \phi\left(\left\|x_{n}-p\right\|\right)+d_{n} \\
& \leq\left\|x_{n}-p\right\|+c_{n}\left[\phi(\beta)+\alpha\left\|x_{n}-p\right\|\right]+d_{n} \\
& =\left(1+\alpha c_{n}\right)\left\|x_{n}-p\right\|+c_{n} \phi(\beta)+d_{n} .
\end{aligned}
$$


Putting $\tilde{c}_{n}:=\alpha c_{n}$ and $\tilde{d}_{n}:=c_{n} \phi(\beta)+d_{n}$, this implies that

$$
\left\|x_{n+1}-p\right\| \leq\left(1+\tilde{c}_{n}\right)\left\|x_{n}-p\right\|+\tilde{d}_{n}
$$

and

$$
\sum_{n=1}^{\infty} \tilde{c}_{n}<\infty \quad \text { and } \quad \sum_{n=1}^{\infty} \tilde{d}_{n}<\infty
$$

by using (A2). So, by Lemma 2.2 , the limit $\lim _{n \rightarrow \infty}\left\|x_{n}-p\right\|$ exists.

Now to show (ii), taking the infimum over all $p \in F$ on the both sides of inequality (2.2) we obtain

$$
d\left(x_{n+1}, F\right) \leq\left(1+\tilde{c}_{n}\right) d\left(x_{n}, F\right)+\tilde{d}_{n} .
$$

Applying Lemma 2.2 again, (ii) is quickly obtained.

Proof of Theorem 2.1. It suffices to show the sufficiency. Assume that

$$
\liminf _{n \rightarrow \infty} d\left(x_{n}, F\right)=0 .
$$

Then it follows from (ii) of Lemma 2.3 that $\lim _{n \rightarrow \infty} d\left(x_{n}, F\right)=0$. Since $\sum \tilde{c}_{n}<\infty$ in the process proving Lemma 2.3 , we observe that

$$
1 \leq K:=\prod\left(1+\tilde{c}_{n}\right) \leq e^{\sum \tilde{c}_{n}}<\infty .
$$

Given $\epsilon>0$, since $\lim _{n \rightarrow \infty} d\left(x_{n}, F\right)=0$ and $\sum \tilde{d}_{n}<\infty$, we can choose a positive integer $n_{0}$ sufficiently large so that

$$
d\left(x_{n}, F\right)<\frac{\epsilon}{4 K} \quad \text { and } \quad \sum_{i=n}^{\infty} \tilde{d}_{i}<\frac{\epsilon}{4 K}, \quad n \geq n_{0} .
$$

Let $n, m \geq n_{0}$ and $p \in F$. First, use the inequality (2.2) repeatedly together with (2.4) to derive

$$
\begin{aligned}
& \left\|x_{n}-p\right\| \\
\leq & \prod_{i=n_{0}}^{n-1}\left(1+\tilde{c}_{i}\right)\left\|x_{n_{0}}-p\right\|+\sum_{i=n_{0}}^{n-2} \tilde{d}_{i} \prod_{k=i+1}^{n-1}\left(1+\tilde{c}_{k}\right)+\tilde{d}_{n-1} \\
\leq & K\left[\left\|x_{n_{0}}-p\right\|+\sum_{i=n_{0}}^{n-1} \tilde{d}_{i}\right],
\end{aligned}
$$


which implies that

$$
\begin{aligned}
& \left\|x_{n}-x_{m}\right\| \leq\left\|x_{n}-p\right\|+\left\|x_{m}-p\right\| \\
\leq & K\left[\left\|x_{n_{0}}-p\right\|+\sum_{i=n_{0}}^{n-1} \tilde{d}_{i}\right]+K\left[\left\|x_{n_{0}}-p\right\|+\sum_{i=n_{0}}^{m-1} \tilde{d}_{i}\right] \\
\leq & 2 K\left[\left\|x_{n_{0}}-p\right\|+\sum_{i=n_{0}}^{\infty} \tilde{d}_{i}\right] .
\end{aligned}
$$

Taking the infimum over all $p \in F$ firstly on both sides and next using (2.5), we have

$$
\begin{aligned}
\left\|x_{n}-x_{m}\right\| & \left.\leq 2 K\left[d\left(x_{n_{0}}, F\right)+\sum_{i=n_{0}}^{\infty} \tilde{d}_{i}\right]\right) \\
& \leq 2 K\left(\frac{\epsilon}{4 K}+\frac{\epsilon}{4 K}\right)=\epsilon, \quad n, m \geq n_{0} .
\end{aligned}
$$

This shows that $\left\{x_{n}\right\}$ is a Cauchy sequence in $X$. Say $x_{n} \rightarrow x^{*} \in X$. Finally, we claim that $x^{*} \in F$. In fact, note first that

$$
\left\|x^{*}-p\right\| \leq\left\|x^{*}-x_{n}\right\|+\left\|x_{n}-p\right\|
$$

for all $p \in F$ and $n \geq 1$. Taking the infimum again over all $p \in F$ on both sides ensures that

$$
d\left(x^{*}, F\right) \leq\left\|x^{*}-x_{n}\right\|+d\left(x_{n}, F\right) \rightarrow 0
$$

as $n \rightarrow \infty$. Since $F$ is closed by continuity of $\mathcal{S}$, it follows that $x^{*} \in F$ and the proof is complete.

Corollary 2.4. Under the same hypotheses as Theorem 2.1, the sequence $\left\{x_{n}\right\}$ converges strongly to a common fixed point $p \in F$ if and only if there exists a subsequence $\left\{x_{n_{k}}\right\}$ of $\left\{x_{n}\right\}$ which converges strongly to $p$.

Proof. Note that there exists a subsequence $\left\{x_{n_{k}}\right\}$ of $\left\{x_{n}\right\}$ which converges strongly to $p$ if and only if $\lim _{k \rightarrow \infty} d\left(x_{n_{k}}, F\right)=0$. Since

$$
\liminf _{n \rightarrow \infty} d\left(x_{n}, F\right) \leq \liminf _{k \rightarrow \infty} d\left(x_{n_{k}}, F\right)=\lim _{k \rightarrow \infty} d\left(x_{n_{k}}, F\right)=0,
$$

it follows that $\liminf _{n \rightarrow \infty} d\left(x_{n}, F\right)=0$. Now apply Theorem 2.1 to complete the proof.

\section{Applications to a finite family of TAN self mappings}

Let $C$ be a nonempty closed convex subset of a real Banach space $X$ and let $N \geq 1$ be fixed. Let $\left\{T_{i}\right\}_{i=1}^{N}$ be a finite family of $N$ continuous TAN mappings defined on $C$, that is, for $i=1,2, \cdots, N$,

$$
\left\|T_{i}^{n} x-T_{i}^{n} y\right\| \leq\|x-y\|+c_{n}^{(i)} \phi_{i}(\|x-y\|)+d_{i}^{(i)}
$$


for all $x, y \in C$, where $\phi_{i} \in \Gamma\left(\mathbb{R}^{+}\right),\left\{c_{n}^{(i)}\right\}$ and $\left\{d_{n}^{(i)}\right\}$ are sequences of nonnegative real numbers such that $c_{n}^{(i)} \rightarrow 0, d_{n}^{(i)} \rightarrow 0$ as $n \rightarrow \infty(1 \leq i \leq N)$.

In this section, as a special case, we recall the following explicit iteration algorithm (1.13) studied by Chidume and Ofoedu [3] for such a finite family $\left\{T_{i}\right\}_{i=1}^{N}$ :

$$
\left\{\begin{array}{l}
x_{1} \in C \text { chosen arbitrarily, } \\
x_{n+1}=\left(1-\alpha_{n}\right) x_{n}+\alpha_{n} T_{1}^{n} y_{1 n}, \\
y_{1 n}=\left(1-\alpha_{n}\right) x_{n}+\alpha_{n} T_{2}^{n} y_{2 n}, \\
\quad \vdots \\
y_{(N-2) n}=\left(1-\alpha_{n}\right) x_{n}+\alpha_{n} T_{N-1}^{n} y_{(N-1) n}, \\
y_{(N-1) n}=\left(1-\alpha_{n}\right) x_{n}+\alpha_{n} T_{N}^{n} x_{n}, \quad n \geq 1
\end{array}\right.
$$

Then the above algorithm (1.13) can be rewritten as a special form of (1.12) equipped with $T_{n}^{(i)}=T_{i}^{n}, \alpha_{n}^{(i)} \equiv \alpha_{n}$ for $1 \leq i \leq N$, namely,

$$
x_{n+1}=S_{n} x_{n}, \quad n \geq 1,
$$

where

$$
\left\{\begin{array}{c}
S_{n}=\left(1-\alpha_{n}\right) I+\alpha_{n} T_{1}^{n} U_{n}^{(1)} \\
U_{n}^{(1)}=\left(1-\alpha_{n}\right) I+\alpha_{n} T_{2}^{n} U_{n}^{(2)} \\
\vdots \\
U_{n}^{(N-2)}=\left(1-\alpha_{n}\right) I+\alpha_{n} T_{N-1}^{n} U_{n}^{(N-1)} \\
U_{n}^{(N-1)}=\left(1-\alpha_{n}\right) I+\alpha_{n} T_{N}^{n}, \quad n \geq 1
\end{array}\right.
$$

$x_{1} \in K$ is arbitrarily given, $\alpha_{n} \in[0,1]$ and $y_{i n}=U_{n}^{(i)} x_{n}$ for $1 \leq i \leq N$.

By virtue of Example 1.7, $\mathcal{S}=\left\{S_{n}: C \rightarrow C\right\}$ is obviously continuous TAN on $C$, and $F_{N}=\cap_{i=1}^{N} F\left(T_{i}\right) \subset F=\cap_{n=1}^{\infty} F\left(S_{n}\right)$.

Corollary 3.1([3]). Let $C$ be a nonempty closed convex subset of a real Banach space $X, N \geq 1$ a positive integer, and let $\left\{T_{i}\right\}_{i=1}^{N}$ be a finite family of TAN mappings from $C$ into itself with $F_{N}=\cap_{i=1}^{N} F\left(T_{i}\right) \neq \emptyset$. Let $\left\{x_{n}\right\}$ be the sequence defined explicitly by (1.13). Assume that $\left\{c_{n}^{(i)}\right\},\left\{d_{n}^{(i)}\right\}$ and $\phi_{i}, 1 \leq i \leq N$, satisfy properties (C1) and (C2). Then $\left\{x_{n}\right\}$ converges strongly to a common fixed point of $\left\{T_{i}\right\}_{i=1}^{N}$ if and only if $\liminf _{n \rightarrow \infty} d\left(x_{n}, F_{N}\right)=0$.

Proof. By Example 1.7, since the family $\mathcal{S}=\left\{S_{n}: C \rightarrow C\right\}$ defined recursively by (3.2) is continuous TAN on $C$ and $F_{N} \subset F$, and $(C 1) \Leftrightarrow(\widetilde{C} 1) \Leftrightarrow(\widetilde{C} 1)^{\prime} \Rightarrow(A 1)$ and $(C 2) \Leftrightarrow(\widetilde{C} 2) \Rightarrow(\widetilde{C} 2)^{\prime} \Leftrightarrow(A 2)$, all the assumptions in Theorem 2.1 are therefore fulfilled. Now to prove the sufficiency, assume that $\liminf _{n \rightarrow \infty} d\left(x_{n}, F_{N}\right)=0$. Since $F_{N} \subset F,(2.2)$ still remains true for all $p \in F_{N}$ and so $\lim _{n \rightarrow \infty} d\left(x_{n}, F_{N}\right)$ exists. Hence $\lim _{n \rightarrow \infty} d\left(x_{n}, F_{N}\right)=0$. Now similarly mimicking the proof of Theorem 2.1 with $F_{N}$ instead $F$, we conclude that $\left\{x_{n}\right\}$ strongly converges to a common fixed point of $\left\{T_{i}\right\}_{i=1}^{N}$. 
Remark 3.2. (a) We still don't know whether $F \subset F_{N}$ under the hypotheses of Corollary 3.1 or not.

(b) Note that our proofs in Theorem 2.1 and Corollary 3.1 are simpler than the one given by Chidume and Ofoedu [3].

\section{References}

[1] Ya. I. Alber, C. E. Chidume and H. Zegeye, Approximating fixed points of total asymptotically nonexpansive mappings, Fixed Point Theory and Appl., 2006(2006), article ID 10673, 20 pages.

[2] R. E. Bruck, T. Kuczumow and S. Reich, Convergence of iterates of asymptotically nonexpansive mappings in Banach spaces with the uniform Opial property, Colloq. Math., 65(1993), 169-179.

[3] C. E. Chidume and E. U. Ofoedu, Approximation of common fixed points for finite families of total asymptotically nonexpansive mappings, J. Math. Anal. Appl., 333(2007), 128-141.

[4] K. Goebel and W. A. Kirk, A fixed point theorem for asymptotically nonexpansive mappings, Proc. Amer. Math. Soc., 35(1972), 171-174.

[5] K. Goebel and W.A. Kirk, Topics in Metric Fixed Point Theory, Cambridge Univ. Press, Cambridge, 1990.

[6] G. E. Kim and T. H. Kim, Mann and Ishikawa iterations with errors for nonLipschitzian mappings in Banach spaces, Comput. Math. Appl., 42(2001), 1565-1570.

[7] W. A. Kirk, Fixed point theorems for non-Lipschitzian mappings of asymptotically nonexpansive type, Israel J. Math., 17(1974), 339-346.

[8] M. O. Osilike, S. C. Aniagbosor, and B. G. Akuchu, Fixed points of asymptotically demicontractive mappings in arbitrary Banach spaces, PanAm. Math. J., 12(2002), $77-88$.

[9] W. Takahashi, Nonlinear Functional Analysis, Yokohama Publishers, Yokohama, 2000.

[10] K. K. Tan and H. K. Xu, Approximating fixed points of nonexpansive mappings by the Ishikawa iteration process, J. Math. Anal. Appl., 178(1993), 301-308. 